\title{
Distributed Integral Action: Stability Analysis and Frequency Control of Power Systems
}

\author{
Martin Andreasson*†, Henrik Sandberg*, Dimos V. Dimarogonas* and Karl H. Johansson* \\ ${ }^{*}$ ACCESS Linnaeus Center, KTH Royal Institute of Technology, Stockholm, Sweden.
}

\begin{abstract}
This paper analyzes distributed proportionalintegral controllers. We prove that integral action can be successfully applied to consensus algorithms, where attenuation of static disturbances is achieved. These control algorithms are applied to decentralized frequency control of electrical power systems. We show that the proposed algorithm can attenuate step disturbances of power loads. We provide simulations of the proposed control algorithm on the IEEE 30 bus test system that demonstrate its efficiency.
\end{abstract}

\section{INTRODUCTION}

Distributed control is in many large-scale systems the only feasible control strategy. To attenuate disturbances, proportional-integral (PI) controllers are often employed in such plants. However, it is still an open problem when distributed controllers can stabilize a plant in general [1].

One important and increasingly interesting class of systems that require integral action to attenuate disturbances are electric power systems. Due to disturbances in form of load and generation changes, a proportional frequency controller is not able to reach the desired reference frequency asymptotically. To eliminate static errors, integrators are employed. Due to the inherent difficulties with distributed PI control, automatic frequency control of power systems is typically carried out at two levels. In the inner control loop the frequency is controlled with a proportional controller against a dynamic reference frequency. At the outer loop, the reference frequency is controlled with a PI controller to eliminate static errors [2]. While this control architecture often works satisfactorily today, future power system developments might render it unsuitable. For instance, large scale penetration of renewable power generation increases generation fluctuations, creating a need for fast and local disturbance attenuation. Decentralized control of power systems might also provide efficient anti-islanding control and self-healing features, even when communication is unavailable [3], [4].

In this paper we propose a fully decentralized frequency control algorithm for electrical power systems. We model the power system by the swing equation, used in transient stability [5] and fault detection [6]. We apply and further develop the theory of distributed consensus to prove the stability of the proposed algorithms. These results could be of interest

This work was supported in part by the European Commission by the Hycon2 project, the Swedish Research Council (VR) and the Knut and Alice Wallenberg Foundation. The $3^{\text {rd }}$ author is also affiliated with the Centre for Autonomous Systems at KTH and is supported by the VR 2009-3948 grant. $\dagger$ Corresponding author. E-mail: mandreas@kth.se not only for power systems, but also for other distributed or decentralized dynamical systems with first- or second-order dynamics. We give a short review of the consensus problem to position our theoretical results with respect to the literature. The coordination of autonomous agents based solely on local interactions and decentralized control algorithms [7], has applications in multi-vehicle control [8], formation-control [9], flocking [10], rendezvous [11] and distributed estimation [12], [13]. Consensus with integral action has been studied in [14] for agents with single integrator dynamics. It was shown that the proposed consensus protocol can attenuate constant and some time-varying disturbances to a certain degree. The main theoretical contributions of this paper is the extension of consensus for agents with single integrator dynamics under integral action, and consensus for agents with damped double integrator dynamics under integral action. We study consensus algorithms both with and without absolute position measurements.

The rest of this paper is organized as follows. In section II we introduce the mathematical notation. In section III we analyze consensus protocols with PI controllers for agents with single integrator dynamics. In section IV we propose two consensus protocols for agents with double integrator dynamics, one without and one with absolute position measurements. We also provide av example to support our theoretical results. In section $\mathrm{V}$, based on the developed theory, we propose a decentralized control algorithm for frequency control of electrical power systems, and compare the performance to traditional control algorithms. The paper ends by some concluding remarks in section VI.

\section{Preliminaries}

Let $\mathcal{G}$ be a graph. Denote by $\mathcal{V}=\{1, \ldots, n\}$ the vertex set of $\mathcal{G}$, and by $\mathcal{E}=\{1, \ldots, m\}$ the edge set of $\mathcal{G}$. Let $\mathcal{N}_{i}$ be the set of neighboring nodes to $i$. Denote by $\mathcal{B}=$ $\mathcal{B}(\mathcal{G})$ the vertex-edge adjacency matrix of $\mathcal{G}$, and let $\mathcal{L}$ be the Laplacian matrix of $\mathcal{G}$. In this paper we will only consider static, undirected and connected graphs. For the application of frequency control of power systems, this is a reasonable assumption as long as there are no power line failures. For undirected graphs it holds that $\mathcal{L}=\mathcal{B B}^{T}$. Let $\mathbb{C}^{-}$denote the open left half complex plane, and $\overline{\mathbb{C}}^{-}$its closure. We will denote the position of agent $i$ as $x_{i}$, and its velocity as $v_{i}$, and collect them into column vectors $x=\left(x_{1}, \ldots, x_{n}\right)^{T}$, $v=\left(v_{1}, \ldots, v_{n}\right)^{T}$. We denote by $c_{n \times m}$ a vector or matrix 
of dimension $n \times m$ whose elements are all $c$. $I_{n}$ denotes the identity matrix of dimension $n$.

\section{CONSENSUS BY DisTRIBUTED INTEGRAL ACTION} FOR SINGLE INTEGRATOR DYNAMICS WITH DAMPING

Consider agents with dynamics:

$$
\begin{aligned}
& \dot{z}_{i}=x_{i} \\
& \dot{x}_{i}=-\sum_{j \in \mathcal{N}_{i}}\left(\alpha\left(z_{i}-z_{j}\right)+\beta\left(x_{i}-x_{j}\right)\right)-\delta\left(x_{i}-x_{i}(0)\right)+d_{i}
\end{aligned}
$$

where $\alpha \in \mathbb{R}^{+}, \beta \in \mathbb{R}^{+}, \delta \in \mathbb{R}^{+}$are fixed parameters, and $d_{i} \in \mathbb{R}$ is an unknown disturbance.

Theorem 1. Under the dynamics (1)-(1) the agents converge to a common value $x^{*}$ for any constant disturbance $d_{i}$. If $d_{i}=0 \forall i \in \mathcal{V}$, the agents converge to $x^{*}=\frac{1}{n} \sum_{i \in \mathcal{V}} x_{i}(0)$ for arbitrary $v_{i}(0)$, and for $z_{i}(0)=0$ foralli $\in \mathcal{V}$. If absolute position measurements are not present, i.e. $\delta=0$, we still have $\lim _{t \rightarrow \infty}\left|x_{i}(t)-x_{j}(t)\right|=0 \forall i, j \in \mathcal{V}$ for any set of disturbances $d_{i}$ and any $\alpha, \beta \in \mathbb{R}^{+}$. However the absolute states are in general unbounded, i.e. $\lim _{t \rightarrow \infty}\left|x_{i}(t)\right|=$ $\infty \forall i, j \in \mathcal{V}$.

Proof: First consider the case where $\delta=0$ and $d_{i}=$ $0 \forall i \in \mathcal{V}$. By introducing $z=\left[z_{1}, \ldots, z_{n}\right]^{T}$ we may rewrite (1) - (2) as:

$$
\left[\begin{array}{c}
\dot{z} \\
\dot{x}
\end{array}\right]=\underbrace{\left[\begin{array}{cc}
0_{n \times n} & I_{n} \\
-\alpha \mathcal{L} & -\beta \mathcal{L}
\end{array}\right]}_{\triangleq A}\left[\begin{array}{l}
z \\
x
\end{array}\right] .
$$

By elementary column operations we note that the characteristic equation of $A$ is given by $0=\operatorname{det}\left((\beta s+\alpha) \mathcal{L}+s^{2} I_{n}\right)$. By comparing the characteristic polynomial with the characteristic equation of $\mathcal{L}$ : $0=\operatorname{det}\left(\mathcal{L}-\kappa I_{n}\right)$, with solutions $\kappa=\lambda_{i} \geq 0$, we obtain the equation $0=s^{2}+\lambda_{i} \beta s+\lambda_{i} \alpha$. This equation has two solutions $s=0$ if $\lambda_{i}=0$, and solutions $s \in \mathbb{C}^{-}$if $\lambda_{i}>0$. Since the above equation has exactly two solutions for every $\lambda_{i}$, it follows that the algebraic multiplicity of the eigenvalue 0 must be two. It is well-known that for connected graphs $\mathcal{G}, \lambda_{1}$ is the only zeroeigenvalue of the Laplacian $\mathcal{L}$. By straightforward calculations we obtain that $e_{1}^{1}=\left[\begin{array}{ll}1_{1 \times n} & 0_{1 \times n}\end{array}\right]^{T}$ is an eigenvector and $e_{1}^{2}=\left[\begin{array}{ll}0_{1 \times n} & 1_{1 \times n}\end{array}\right]^{T}$ is a generalized eigenvector of $A$ corresponding to the eigenvalue 0 . It can also be verified that $v_{1}=\frac{1}{n}\left[\begin{array}{ll}1_{1 \times n} & 0_{1 \times n}\end{array}\right]$ and $v_{2}=\frac{1}{n}\left[\begin{array}{ll}0_{1 \times n} & 1_{1 \times n}\end{array}\right]$ are a generalized left eigenvector and an eigenvector of $A$, respectively, corresponding to the eigenvalue 0 , and that $v_{1} e_{1}^{1}=1, v_{2} e_{1}^{2}=1$ and $v_{2} e_{1}^{1}=0, v_{1} e_{1}^{2}=0$. If we let $P$ be an orthonormal matrix consisting of the normalized eigenvectors of $A$, we can chose the first columns of $P$ to be $e_{1}^{1}$ and $e_{1}^{2}$, and the first rows of $P^{-1}$ to be $v_{1}$ and $v_{2}$, respectively. Since all other eigenvalues of $A$ have strictly negative real part we obtain

$$
\begin{aligned}
& \lim _{t \rightarrow \infty} e^{A t}=\lim _{t \rightarrow \infty} P e^{J t} P^{-1} \\
& =P \lim _{t \rightarrow \infty}\left[\begin{array}{ccc}
1 & t & 0_{1 \times(2 n-2)} \\
0 & 1 & 0_{1 \times(2 n-2)} \\
0_{(2 n-2) \times 1} & 0_{(2 n-2) \times 1} & e^{J^{\prime} t}
\end{array}\right] P^{-1} \\
& =\lim _{t \rightarrow \infty} P\left[\begin{array}{ccc}
1 & t & 0_{1 \times(2 n-2)} \\
0 & 1 & 0_{1 \times(2 n-2)} \\
0_{(2 n-2) \times 1} & 0_{(2 n-2) \times 1} & 0_{(2 n-2) \times(2 n-2)}
\end{array}\right] P^{-1} \\
& =\lim _{t \rightarrow \infty} \frac{1}{n}\left[\begin{array}{cc}
1_{n \times n} & t 1_{n \times n} \\
0_{n \times n} & 1_{n \times n}
\end{array}\right]
\end{aligned}
$$

Given an initial position $x(0)=x_{0}$, we obtain $\lim _{t \rightarrow \infty} x_{i}(t)=\frac{1}{n} \sum_{i \in \mathcal{V}} x_{0, i} \quad \forall i \in \mathcal{V}$, i.e., the agents converge to the average of their initial positions.

We now consider the case where $\delta=0$ and $d_{i} \neq 0 \forall i \in \mathcal{V}$. Define the output of the system

$$
\left[\begin{array}{l}
y_{z} \\
y_{x}
\end{array}\right]=\underbrace{\left[\begin{array}{cc}
\mathcal{B}^{T} & 0_{(n-1) \times n} \\
0_{(n-1) \times n} & \mathcal{B}^{T}
\end{array}\right]}_{\triangleq C}\left[\begin{array}{l}
z \\
x
\end{array}\right]
$$

and consider the linear coordinate change $x=\hat{S} u, u=\hat{S}^{T} x$, $z=\hat{S} w, w=\hat{S}^{T} z$, where $\hat{S}=\left[\begin{array}{ll}\frac{1}{\sqrt{n}} 1^{n \times 1} & S\end{array}\right]$, and $S$ is a matrix such that $\hat{S}$ is an orthonormal matrix. In the new coordinates, the system dynamics (3) become:

$$
\begin{aligned}
\dot{w} & =u \\
\dot{u} & =\left[\begin{array}{cc}
0 & 0_{1 \times(n-1)} \\
0_{(n-1) \times 1} & -\alpha S^{T} \mathcal{L} S
\end{array}\right] w \\
& +\left[\begin{array}{cc}
0 & 0_{1 \times(n-1)} \\
0_{(n-1) \times 1} & -\beta S^{T} \mathcal{L} S
\end{array}\right] u+\left[\begin{array}{c}
\frac{1}{n} 1_{1 \times(n)} \\
S^{T}
\end{array}\right] d .
\end{aligned}
$$

We note that the states $u_{1}$ and $w_{1}$ are both unobservable and uncontrollable. We thus omit these states to obtain a minimal realization by defining the new coordinates $u^{\prime}=$ $\left[u_{2}, \ldots, u_{n}\right]^{T}$ and $w^{\prime}=\left[w_{2}, \ldots, w_{n}\right]^{T}$, thus obtaining the system dynamics

$$
\left[\begin{array}{c}
\dot{w}^{\prime} \\
\dot{u}^{\prime}
\end{array}\right]=\left[\begin{array}{cc}
0^{(n-1) \times(n-1)} & I_{(n-1)} \\
-\alpha S^{T} \mathcal{L} S & -\beta S^{T} \mathcal{L} S
\end{array}\right]\left[\begin{array}{c}
w^{\prime} \\
u^{\prime}
\end{array}\right]+\left[\begin{array}{c}
S^{T} d \\
0_{(n-1) \times 1}
\end{array}\right] .
$$

By Lemma 10 in [14], $S^{T} \mathcal{L} S$ is invertible, so we may define

$$
\left[\begin{array}{l}
w^{\prime \prime} \\
u^{\prime \prime}
\end{array}\right]=\left[\begin{array}{l}
w^{\prime} \\
u^{\prime}
\end{array}\right]-\left[\begin{array}{c}
\frac{1}{\alpha}\left(S^{T} \mathcal{L} S\right)^{-1} S^{T} d \\
0_{(n-1) \times 1}
\end{array}\right] .
$$

It is easily verified that the origin is the only equilibrium of the system dynamics, which in the new coordinates are given by

$$
\left[\begin{array}{c}
\dot{w}^{\prime \prime} \\
\dot{u}^{\prime \prime}
\end{array}\right]=\underbrace{\left[\begin{array}{cc}
0_{(n-1) \times(n-1)} & I_{(n-1)} \\
-\alpha S^{T} \mathcal{L} S & -\beta S^{T} \mathcal{L} S
\end{array}\right]}_{\triangleq A^{\prime \prime}}\left[\begin{array}{l}
w^{\prime \prime} \\
u^{\prime \prime}
\end{array}\right] .
$$


By [15], the characteristic polynomial in $\kappa$ of $A^{\prime \prime}$ is given by $\operatorname{det}\left(\kappa^{2} I_{(n-1)}+(\beta \kappa+\alpha) S^{T} \mathcal{L} S\right)$. By comparing this polynomial with the characteristic polynomial $\operatorname{det}\left(s I+B^{T} \mathcal{L} B\right)$, which by lemma 10 in [14] has solutions $-s_{i}<0$, we know that the eigenvalues of $A^{\prime \prime}$ must satisfy $\kappa^{2}+s_{i} \beta \kappa+s_{i} \alpha=0$, with solutions $\kappa \in \mathbb{C}^{-}$. Thus $A^{\prime \prime}$ is Hurwitz.

Now consider the case where $\delta \neq 0$ and $d_{i}=0 \forall i \in \mathcal{V}$. The dynamics can be written as:

$$
\left[\begin{array}{c}
\dot{z} \\
\dot{x}
\end{array}\right]=\underbrace{\left[\begin{array}{cc}
0_{n \times n} & I_{n} \\
-\alpha \mathcal{L} & -\beta \mathcal{L}-\delta I
\end{array}\right]}_{\triangleq A}\left[\begin{array}{l}
z \\
x
\end{array}\right] .
$$

By elementary column operations, the characteristic polynomial of $A$ may be written as $0=\operatorname{det}\left((\beta s+\alpha) \mathcal{L}+\left(s^{2}+\delta s\right) I_{n}\right)$. By similar arguments used in the previous parts of the proof, $A$ has a simple eigenvalue 0 , with the corresponding eigenvector $e_{1}=\left[\begin{array}{ll}1_{1 \times n} & 0_{1 \times n}\end{array}\right]^{T}$ and the left eigenvector $v_{1}=\frac{1}{n}\left[\begin{array}{ll}1 \times n & 0_{1 \times n}\end{array}\right]$, whereas all other eigenvalues have negative real part. We see that $v_{1} e_{1}=1$, and hence it follows that

$$
\begin{aligned}
& \lim _{t \rightarrow \infty} e^{A t}=\lim _{t \rightarrow \infty} P e^{J t} P^{-1} \\
& =P \lim _{t \rightarrow \infty}\left[\begin{array}{cc}
1 & 0_{1 \times(2 n-1)} \\
0_{(2 n-1) \times 1} & e^{J^{\prime} t}
\end{array}\right] P^{-1}=\frac{1}{n}\left[\begin{array}{cc}
1_{n \times n} & 0_{n \times n} \\
0_{n \times n} & 0_{n \times n}
\end{array}\right]
\end{aligned}
$$

Given any initial position $x(0)=x_{0}$, it immediately follows that $\lim _{t \rightarrow \infty} x(t)=0$. Now let $d_{i} \neq 0$ and $x(0) \neq 0$ for at least one $i \in \mathcal{V}$. Defining the output as

$$
\left[\begin{array}{l}
y_{z} \\
y_{x}
\end{array}\right]=\underbrace{\left[\begin{array}{cc}
\mathcal{B}^{T} & 0_{(n-1) \times n} \\
0_{(n-1) \times n} & I_{n}
\end{array}\right]}_{\triangleq C}\left[\begin{array}{l}
z \\
x
\end{array}\right],
$$

the proof is analogue to the proof when $d_{i} \neq 0$ and $x(0)=$ $0 \forall i \in \mathcal{V}$, and is hence omitted.

Finally, if $d_{i}=0 \forall i \in \mathcal{V}$, the stationarity of $x(t)$ implies $\lim _{t \rightarrow \infty} 1_{1 \times n}(-\alpha \mathcal{L} z(t)-\beta \mathcal{L} x(t)-\delta x(t)+\delta x(0))=0$, so $n x^{*}=\sum_{i \in \mathcal{V}} x_{i}(0)$, which concludes the proof.

Note 1. Theorem 1 guarantees that the agents converge to a common state, albeit constant disturbances. If at least one absolute position measurement is available, the agents converge to a constant, bounded state. Since the agents converge to the average of their initial states in the absence of disturbances, and the system remains stable for any integral gain, there are no immediate performance degradations by the introduction of integral action.

\section{CONSENSUS By DISTRIBUTED INTEGRAL ACTION FOR} DOUBLE INTEGRATOR DYNAMICS WITH DAMPING

In this section we generalize the results of Section III to double integrator dynamics. Consider agents with dynamics:

$$
\begin{aligned}
\dot{z}_{i} & =x_{i} \\
\dot{x}_{i} & =v_{i} \\
\dot{v}_{i} & =-\sum_{j \in \mathcal{N}_{i}}\left(\alpha\left(z_{i}-z_{j}\right)+\beta\left(x_{i}-x_{j}\right)\right)-\gamma v_{i}-\delta\left(x_{i}-x_{i}^{0}\right)+d_{i}
\end{aligned}
$$

where $x_{i}^{0}=x_{i}(0), \alpha \in \mathbb{R}^{+}, \beta \in \mathbb{R}^{+}, \gamma \in \mathbb{R}^{+}, \delta \in \mathbb{R}^{+}$ and $d_{i} \in \mathbb{R}$ is an unknown scalar disturbance. The above protocol does not require communication of the integral state between the agents, as it suffices for each agent to measure its neighbors states and integrate the relative differences.

Theorem 2. Under the dynamics (4)-(6), the agents converge to a common value $x^{*}$ for any constant disturbance $d_{i}$, provided that $\alpha<\beta \gamma$. If $d_{i}=0 \forall i \in \mathcal{V}$, the agents converge to $x^{*}=\frac{1}{n} \sum_{i \in \mathcal{V}} x_{i}(0)$ for arbitrary $v_{i}(0)$. If absolute position measurements are not present, i.e., $\delta=0$, we still have $\lim _{t \rightarrow \infty}\left|x_{i}(t)-x_{j}(t)\right|=0 \forall i, j \in \mathcal{V}$ for any set of disturbances $d_{i}$. However the absolute states are in general unbounded, i.e., $\lim _{t \rightarrow \infty}\left|x_{i}(t)\right|=\infty \forall i, j \in \mathcal{V}$. Also, the system is stable if and only if $\alpha<\beta \gamma$.

Proof: The proof follows the same principle ideas as the proof of Theorem 1. However, as we consider second-order dynamics, the problem is inherently different to first-order dynamics. First consider the case where $\delta=0$. We first let $\delta_{i}=0 \forall i \in \mathcal{V}$. Let also $d_{i}=0 \forall i \in \mathcal{V}$. By introducing the state vector $z=\left[z_{1}, \ldots, z_{n}\right]^{T}$ we may rewrite $(4)-(6)$ in vector form as:

$$
\left[\begin{array}{c}
\dot{z} \\
\dot{x} \\
\dot{v}
\end{array}\right]=\underbrace{\left[\begin{array}{ccc}
0_{n \times n} & I_{n} & 0_{n \times n} \\
0_{n \times n} & 0_{n \times n} & I_{n} \\
-\alpha \mathcal{L} & -\beta \mathcal{L} & -\gamma I_{n}
\end{array}\right]}_{\triangleq A}\left[\begin{array}{c}
z \\
x \\
v
\end{array}\right] .
$$

By elementary column operations it is easily shown that the characteristic polynomial of $A$ can be written as $0=$ $\operatorname{det}\left((\alpha+\beta s) L+s^{2}(s+\gamma) I\right)$, where $I$ is the identity matrix of appropriate dimensions. Comparing the above equation with the characteristic polynomial of $\mathcal{L}$, we get that $0=s^{3}+$ $\gamma s^{2}+\lambda_{i} \beta s+\lambda_{i} \alpha$, where $\lambda_{i}$ is an eigenvalue of $\mathcal{L}$. If $\lambda_{i}>0$, the above equation has all its solutions $s \in \mathbb{C}^{-}$if and only if $\alpha<\beta \gamma$, and $\alpha, \beta, \gamma>0$ by the Routh-Hurwitz stability criterion. Since $\mathcal{G}$ by assumption is connected, $\lambda_{1}=0$ and $\lambda_{i}>0 \forall i=2, \ldots, n$. For $\lambda_{1}=0$, the above equation has the solutions $s=0, s=-\gamma$. By straightforward calculations it can be shown that $e_{1}^{1}=\left[\begin{array}{lll}1_{1 \times n} & 0_{1 \times n} & 0_{1 \times n}\end{array}\right]^{T}$ and $e_{1}^{2}=$ $\left[\begin{array}{lll}0_{1 \times n} & 1_{1 \times n} & 0_{1 \times n}\end{array}\right]^{T}$ are an eigenvector and a generalized eigenvector, respectively, corresponding to the eigenvalue 0 . Furthermore $v_{1}=\frac{1}{\gamma^{2} n}\left[\begin{array}{lll}\gamma^{2} 1_{1 \times n} & 0_{1 \times n} & 1_{1 \times n}\end{array}\right]$ and $v_{2}=$ $\frac{1}{\gamma n}\left[\begin{array}{lll}0_{1 \times n} & \gamma 1_{1 \times n} & 1_{1 \times n}\end{array}\right]$ are a generalized left eigenvector and a left eigenvector of $A$ corresponding to he eigenvalue 0 . Furthermore $v_{1} e_{1}^{1}=1, v_{2} e_{1}^{2}=1$ and $v_{2} e_{1}^{1}=0, v_{1} e_{1}^{2}=0$. Hence the first columns of $P$ can be chosen as $e_{1}^{1}$ and $e_{1}^{2}$, 
and the first rows of $P^{-1}$ can be chosen to be $v_{1}$ and $v_{2}$. Since all other eigenvalues of $A$ have strictly negative real part we obtain

$$
\begin{aligned}
& \lim _{t \rightarrow \infty} e^{A t}=\lim _{t \rightarrow \infty} P e^{J t} P^{-1} \\
& =\lim _{t \rightarrow \infty} \frac{1}{n}\left[\begin{array}{ccc}
1_{n \times n} & t 1_{n \times n} & \frac{1+t \gamma}{\gamma^{2}} 1_{n \times n} \\
0_{n \times n} & 1_{n \times n} & \frac{1}{\gamma} 1_{n \times n} \\
0_{n \times n} & 0_{n \times n} & 0_{n \times n}
\end{array}\right] .
\end{aligned}
$$

Given any initial position $x(0)=x_{0}, v(0)=v_{0}$, we obtain $\lim _{t \rightarrow \infty} x_{i}(t)=\frac{1}{n} \sum_{i \in \mathcal{V}} x_{0, i}+\frac{1}{\gamma n} \sum_{i \in \mathcal{V}} v_{0, i} \quad \forall i \in \mathcal{V}$. Now let us turn our attention to the case where where $\delta=0$ and $d_{i} \neq 0 \forall i \in \mathcal{V}$. We define the output of the system as

$$
\left[\begin{array}{l}
y_{z} \\
y_{x} \\
y_{v}
\end{array}\right]=\underbrace{\left[\begin{array}{ccc}
\mathcal{B}^{T} & 0_{(n-1) \times n} & 0_{(n-1) \times n} \\
0_{(n-1) \times n} & \mathcal{B}^{T} & 0_{(n-1) \times n} \\
0_{(n-1) \times n} & 0_{(n-1) \times n} & \mathcal{B}^{T}
\end{array}\right]}_{\triangleq C}\left[\begin{array}{l}
z \\
x \\
v
\end{array}\right],
$$

and consider the same linear coordinate change of $z, x$ and $v$ as applied to $z$ and $x$ in the proof of Theorem 1. In the new coordinates the system dynamics are

$$
\begin{aligned}
\dot{z}^{\prime} & =x^{\prime} \\
\dot{x}^{\prime} & =v^{\prime} \\
\dot{v}^{\prime} & =\left[\begin{array}{ll}
0 & 0_{1 \times(n-1)} 0^{(n-1) \times 1}-\alpha S^{T} \mathcal{L} S
\end{array}\right] z^{\prime} \\
& \times\left[\begin{array}{cc}
0 & 0_{1 \times(n-1)} \\
0^{(n-1) \times 1} & -\beta S^{T} \mathcal{L} S
\end{array}\right] x^{\prime}-\gamma v^{\prime}+\left[\begin{array}{c}
\frac{1}{n} 1_{1 \times(n)} \\
S^{T}
\end{array}\right] d .
\end{aligned}
$$

We note that the states $z_{1}^{\prime}, x_{1}^{\prime}$ and $v_{1}^{\prime}$ are unobservable and uncontrollable. We thus omit these states to obtain a minimal realization by defining the new coordinates $z^{\prime \prime}=$ $\left[z_{2}^{\prime}, \ldots, z_{n}^{\prime}\right]^{T}, x^{\prime \prime}=\left[x_{2}^{\prime}, \ldots, x_{n}^{\prime}\right]^{T}$ and $v^{\prime \prime}=\left[v_{2}^{\prime}, \ldots, v_{n}^{\prime}\right]^{T}$ we obtain the system dynamics

$$
\left[\begin{array}{c}
\dot{z}^{\prime \prime} \\
\dot{x}^{\prime \prime} \\
\dot{v}^{\prime \prime}
\end{array}\right]=\underbrace{\left[\begin{array}{ccc}
0_{(n-1)^{2}} & I_{(n-1)^{2}} & 0_{(n-1)^{2}} \\
0_{(n-1)^{2}} & 0_{(n-1)^{2}} & I_{(n-1)^{2}} \\
-\alpha S^{T} \mathcal{L} S & -\beta S^{T} \mathcal{L} S & -\gamma I_{(n-1)^{2}}
\end{array}\right]}_{\triangleq A^{\prime \prime}}\left[\begin{array}{c}
z^{\prime \prime} \\
x^{\prime \prime} \\
v^{\prime \prime}
\end{array}\right]+\left[\begin{array}{c}
S^{T} d \\
0 \\
0
\end{array}\right] .
$$

We now shift the state space by defining

$$
\left[\begin{array}{l}
z^{(3)} \\
x^{(3)} \\
v^{(3)}
\end{array}\right]=\left[\begin{array}{c}
z^{\prime \prime} \\
x^{\prime \prime} \\
v^{\prime \prime}
\end{array}\right]-\left[\begin{array}{c}
\frac{1}{\alpha}\left(S^{T} \mathcal{L} S\right)^{-1} S^{T} d \\
0_{(n-1) \times 1} \\
0_{(n-1) \times 1}
\end{array}\right] .
$$

It is easily verified that the origin is the only equilibrium of the system dynamics, and that the dynamics in the new coordinates are also characterized by the matrix $A^{\prime \prime}$. By a similar argument used when showing that $A$ is negative semi-definite, we may show that $A^{\prime \prime}$ is negative semi-definite. But since $S^{T} \mathcal{L} S$ is full-rank, $A^{\prime \prime}$ must also be full-rank, and hence $A^{\prime \prime}$ is Hurwitz. This implies that $\lim _{t \rightarrow \infty}\left|x_{i}(t)-x_{j}(t)\right|=0 \forall i, j \in \mathcal{V}$ even in the presence of disturbances $d_{i}$. It is also clear that whenever $\alpha \geq \beta \gamma$, at least one eigenvalue will have non-negative real part, and that its (generalized) eigenvector will be distinct from $e_{1}^{1}$ and $e_{1}^{2}$. Thus consensus is not reached.

We now turn our attention to the case where $\delta \neq 0$ and $d_{i}=0 \forall i \in \mathcal{V}$. The dynamics (4)-(6) can then be written in vector form as

$$
\left[\begin{array}{c}
\dot{z} \\
\dot{x} \\
\dot{v}
\end{array}\right]=\underbrace{\left[\begin{array}{ccc}
0_{n \times n} & I_{n} & 0_{n \times n} \\
0_{n \times n} & 0_{n \times n} & I_{n} \\
-\alpha \mathcal{L} & -\beta \mathcal{L}-\delta I & -\gamma I_{n}
\end{array}\right]}_{\triangleq A}\left[\begin{array}{c}
z \\
x \\
v
\end{array}\right] .
$$

By performing elementary column operations the characteristic polynomial of $A$ can be written as $\operatorname{det}(s I) \operatorname{det}((s+$ $\gamma) I) \operatorname{det}\left((\alpha+\beta s) L+\left(s^{3}+\gamma s^{2}+\delta s\right) I\right)$. The first two factors give the solutions $s=0$ and $s=-\gamma$, respectively. By comparing the third factor with the characteristic polynomial of $\mathcal{L}$, it can be seen that the other eigenvalues $s$ of $A$ satisfy $0=s^{3}+\gamma s^{2}+\left(\delta+\lambda_{i} \beta\right) s+\lambda_{i} \alpha$, where $\lambda_{i} \in \operatorname{spec}(\mathcal{L})$ Since $\mathcal{G}$ by assumption is connected, $\mathcal{L}$ has a single simple eigenvalue $\lambda_{1}=0$, which gives the characteristic equation $0=s\left(s^{2}+\gamma s+\delta\right)$, with one solution $s=0$, and two solutions $s \in \mathbb{C}^{-}$. As all other eigenvalues are strictly positive, the solutions $s$ corresponding to strictly positive $\lambda_{i}$ satisfy $s \in \mathbb{C}^{-}$iff $\lambda_{i} \alpha+\delta<\lambda_{i} \beta \gamma$. This is satisfied whenever $\alpha<\beta \gamma$. It can be verified that $e_{1}=\left[\begin{array}{lll}1_{1 \times n} & 0_{1 \times n} & 0_{1 \times n}\end{array}\right]^{T}$ and $v_{1}=\frac{1}{n}\left[\begin{array}{lll}\delta 1_{1 \times n} & \gamma 1_{1 \times n} & 1_{1 \times n}\end{array}\right]^{T}$ are a right and left eigenvector of $A$, corresponding to the eigenvalue 0 . Furthermore $v_{1} e_{1}=1$. Since all other eigenvalues have strictly negative real part, we have

$\lim _{t \rightarrow \infty} e^{A t}=\lim _{t \rightarrow \infty} P e^{J t} P^{-1}=\frac{1}{n}\left[\begin{array}{ccc}\delta 1_{n \times n} & \gamma 1_{n \times n} & 1_{n \times n} \\ 0_{n \times n} & 0_{n \times n} & 0_{n \times n} \\ 0_{n \times n} & 0_{n \times n} & 0_{n \times n}\end{array}\right]$.

Given any initial position $x(0)=x_{0}, v(0)=v_{0}$, we obtain that $\lim _{t \rightarrow \infty} x(t)=0$. Now when $\delta \neq 0$ and $d_{i} \neq 0$ for at least one $i \in \mathcal{V}$, defining the output of the system as

$$
\left[\begin{array}{l}
y_{z} \\
y_{x} \\
y_{v}
\end{array}\right]=\underbrace{\left[\begin{array}{ccc}
\mathcal{B}^{T} & 0_{(n-1) \times n} & 0_{(n-1) \times n} \\
0_{(n-1) \times n} & \mathcal{B}^{T} & 0_{(n-1) \times n} \\
0_{n \times n} & 0_{n \times n} & I_{n}
\end{array}\right]}_{\triangleq_{C}}\left[\begin{array}{l}
z \\
x \\
v
\end{array}\right]
$$

the proof is analogous to the proof when $\delta=0$ and $d_{i} \neq$ $0 \forall i \in \mathcal{V}$, and is hence omitted.

Finally, if $d_{i}=0 \forall i \in \mathcal{V}$, the stationarity of $v(t)$ implies: $\lim _{t \rightarrow \infty} 1_{1 \times n}(-\alpha \mathcal{L} z-\beta \mathcal{L} x-\delta x+\delta x(0)-\gamma v)=0$, so $n x^{*}=\sum_{i \in \mathcal{V}} x_{i}(0)$, which concludes the proof.

Example 1. In this example we will consider the task of reaching position-consensus for a multi-robot system. We consider a distributed PI controller for mobile robots with velocity-dependent damping of the form of (4)-(6). Let the parameters be given by $\beta=5, \gamma=3$ and $\delta=0$. The setup we will consider consists of a string of 5 mobile robots, whose communication topology is a string graph. We consider the system with a constant disturbance $d=[1,0,0,0,0]$, for $\alpha=0, \alpha=1$, and $\alpha=15$. The initial conditions are given by $x(0)=[5,-6,8,4,5]^{T}, v(0)=[0,0,0,0,0]^{T}$. 

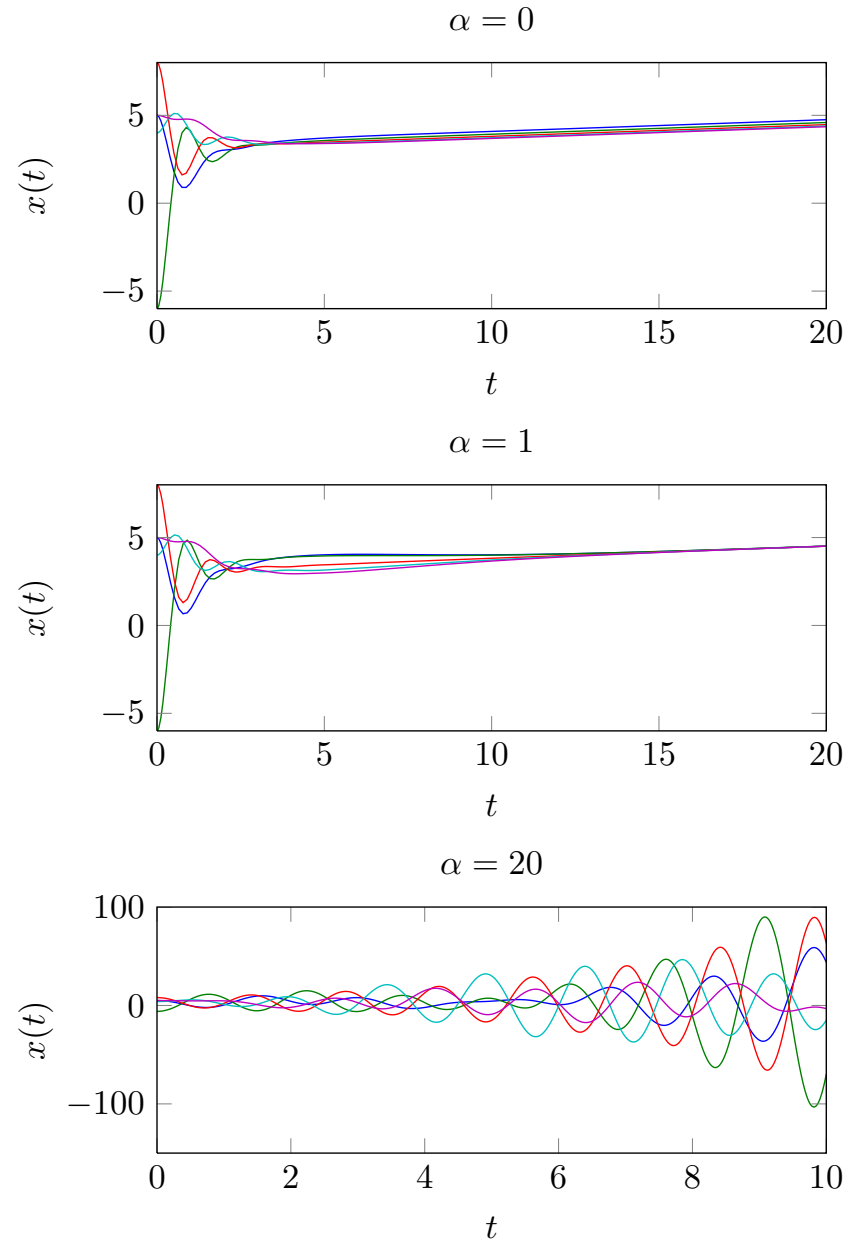

Figure 1. The first figure shows the state trajectories of (4)-(6) when $\alpha=0$, the second figure shows the state trajectories when $\alpha=1$, the third figure shows the state trajectories when $\alpha=20$.

By Theorem 2 stability is guaranteed if and only if $\alpha<$ $\beta \gamma$. In Figure 1 the state trajectories are shown for different choices of $\alpha$. We observe that asymptotic consensus amongst the mobile robots is only reached when $\alpha=1$. When $\alpha=$ 0 , consensus is not reached due to the static disturbance. When $\alpha=1$, the disturbance is attenuated, and asymptotic consensus is reached. However, as we increase $\alpha$ to $20=\beta \gamma$, the system becomes unstable, in accordance with Theorem 2. This is illustrated in the lower right plot of Figure 1.

\section{FREQUENCY CONTROL OF POWER SYSTEMS}

In this section we show that a similar protocol to the one proposed in section IV, can be employed for automatic frequency control in power systems. Let us consider a power system modeled by a graph $\mathcal{G}=(\mathcal{V}, \mathcal{E})$. Each node, here referred to as a bus, is assumed to obey the linearized swing equation [2]

$$
m_{i} \ddot{\delta}_{i}+d_{i} \dot{\delta}_{i}=-\sum_{j \in \mathcal{N}_{i}} k_{i j}\left(\delta_{i}-\delta_{j}\right)+p_{i}^{m}+u_{i}
$$

where $\delta_{i}$ is the phase angle of bus $i, m_{i}$ and $d_{i}$ are the inertia and damping coefficient respectively, $p_{i}^{m}$ is the power load at bus $i$ and $u_{i}$ is the mechanical input. $k_{i j}=\left|V_{i}\right|\left|V_{j}\right| b_{i j}$, where $V_{i}$ is the voltage of bus $i$, and $b_{i j}$ is the susceptance of the edge $(i, j)$, here referred to as line. By defining the state vectors $\delta=\left[\delta_{i}, \ldots, \delta_{n}\right]$ and $\omega=\dot{\delta}=\left[\omega_{1} \ldots, \omega_{n}\right]$, we may rewrite (7) in state-space form as

$$
\left[\begin{array}{c}
\dot{\delta} \\
\dot{\omega}
\end{array}\right]=\left[\begin{array}{cc}
0_{n \times n} & I_{n} \\
-M \mathcal{L}_{k} & -M D
\end{array}\right]\left[\begin{array}{c}
\delta \\
\omega
\end{array}\right]+\left[\begin{array}{c}
0_{n \times 1} \\
M p^{m}
\end{array}\right]+\left[\begin{array}{c}
0_{n \times 1} \\
M u
\end{array}\right]
$$

where $M=\operatorname{diag}\left(\frac{1}{m_{1}}, \ldots, \frac{1}{m_{n}}\right), D=\operatorname{diag}\left(d_{1}, \ldots, d_{n}\right), \mathcal{L}_{k}$ is the weighted Laplacian with edge weights $k_{i j}, p^{m}=$ $\left[p_{1}^{m}, \ldots, p_{n}^{m}\right]^{T}, u=\left[u_{i}, \ldots, u_{n}\right]^{T}$.

\section{A. Centralized PI control}

Traditionally, automatic frequency control of power systems is carried out at two levels [2]. In the inner control loop the frequency is controlled with a proportional controller against a reference frequency. At the outer loop, the reference frequency is controlled with a PI controller to eliminate static errors. We model the inner controller of bus $i$ as

$$
u_{i}=\alpha\left(\hat{\omega}-\omega_{i}\right)
$$

and the outer PI controller is assumed to be given by

$$
\begin{aligned}
& \dot{\hat{z}}=\omega^{\text {ref }}-\frac{1}{n} \sum_{i \in \mathcal{V}} \omega_{i} \\
& \dot{\hat{\omega}}=\omega^{\text {ref }}-\frac{1}{n} \sum_{i \in \mathcal{V}} \omega_{i}+\beta \hat{z}
\end{aligned}
$$

where we have assumed that the average frequency of the buses is measured by the central controller. In reality the frequency is often measured at a specific bus. This will typically lead to longer delays, since disturbances need to propagate through the system before control action can be taken. The centralized controller architecture is illustrated in figure 2 .

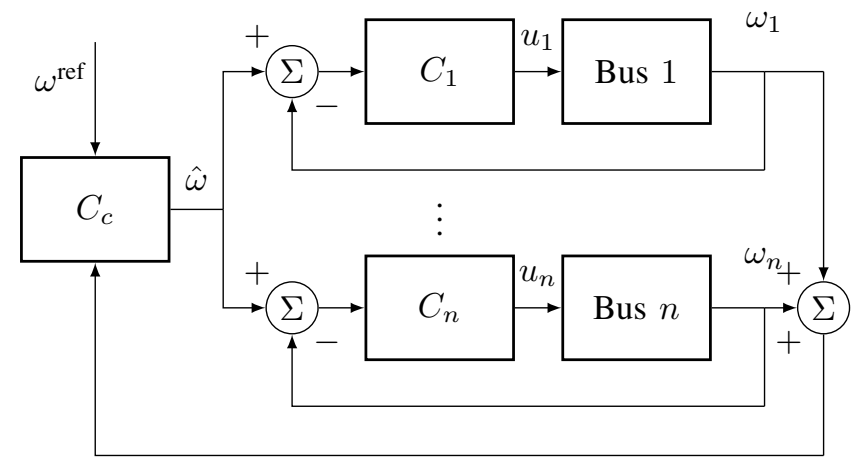

Figure 2. The figure illustrates the centralized control architecture.

Theorem 3. The power system described by (8) where $u_{i}$ is given by (9)-(11), is stable for $\alpha>0, \beta>0, \gamma>0$ satisfying $\beta \gamma<\alpha$. Furthermore $\lim _{t \rightarrow \infty} \omega_{i}(t)=\omega^{\text {ref }}$. 


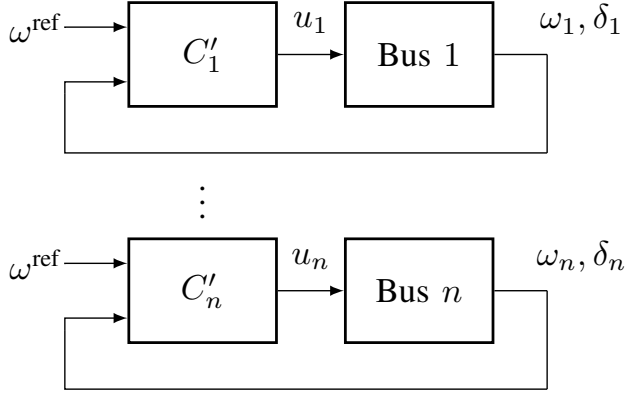

Figure 3. The figure illustrates the decentralized control architecture.

Proof: As we are only interested in studying $\omega$, we may rewrite (8) with $u$ given by (9)-(11), as

$$
\left[\begin{array}{c}
\dot{\hat{\omega}} \\
\dot{z} \\
\dot{\omega}
\end{array}\right]=\underbrace{\left[\begin{array}{ccc}
0 & -\frac{\beta}{n} 1_{1 \times n} & -\frac{\gamma}{n} 1_{1 \times n} \\
0_{n \times 1} & 0_{n \times n} & I_{n} \\
\alpha 1_{n \times 1} & -M \mathcal{L} & -M D-\alpha I_{n}
\end{array}\right]}_{\triangleq A} \cdot\left[\begin{array}{c}
\hat{\omega} \\
z \\
\omega
\end{array}\right]+\left[\begin{array}{c}
\gamma \omega^{\mathrm{ref}} \\
-\omega^{\mathrm{ref}} 1 \\
p^{m}
\end{array}\right]
$$

We now consider the the matrix $A^{\prime}$ defined as

$$
A^{\prime} \triangleq\left[\begin{array}{ccc}
0 & -\frac{\beta}{n} 1_{1 \times n} & -\frac{\gamma}{n} 1_{1 \times n} \\
0_{n \times 1} & 0_{n \times n} & I_{n} \\
\alpha 1_{n \times 1} & -M \mathcal{L} & -\alpha I_{n}
\end{array}\right]
$$

By performing elementary column operations on $A^{\prime}$, we may write the characteristic polynomial of $A^{\prime}$ as $\left(s^{3} \gamma+s^{2} \alpha+s \alpha+\alpha \beta\right) \cdot \operatorname{det}(M \mathcal{L}+(s+\alpha))=0$. The first factor has solutions $s \in \mathbb{C}^{-}$if $\beta \gamma<\alpha$. Comparing the second factor with the characteristic polynomial of $M \mathcal{L}$, we have that $s$ must satisfy $s^{2}+s \alpha+t_{i}=0$, where $t_{i} \geq 0$ is an eigenvalue of $M \mathcal{L}$. Also this equation has all solutions $s \in \mathbb{C}^{-}$. Thus $A$ must be Hurwitz. Finally, stationarity implies that the equilibrium solution must satisfy $\omega=\omega^{\text {ref }} 1_{n \times 1}$.

\section{B. Decentralized PI control}

In this section we analyze a completely decentralized PI controller, where each bus controls its own frequency based only on local information. Thus, no frequency measurements need to be sent to a central controller, and there is no need to send control signals. This architecture might be favorable due to security concerns when sending unencrypted frequency measurements and control signals over large areas. Another benefit could be better performance when the tripping of one or several power lines causes the network to be split up into two or more sub-networks, so called islanding. The controller of node $i$ is assumed to be given by

$$
\begin{aligned}
\dot{z}_{i} & =\left(\omega^{\text {ref }}-\omega_{i}\right) \\
u_{i} & =\alpha\left(\omega^{\text {ref }}-\omega_{i}\right)+\beta z_{i}
\end{aligned}
$$

The controller architecture is illustrated in Figure 3. This decentralized control architecture is not practically feasible with only frequency measurements available at the generation buses. Even the slightest frequency measurement error will be integrated and prevent the frequencies from reaching consensus [2]. With recent advances in phasor measurement unit (PMU) technology however, phase measurements are likely to be available to all generator buses [16]. By employing optimal PMU placement, the number of PMU:s needed for complete observability can be drastically reduced [17]. By integrating (13) we obtain $z_{i}=\omega^{\text {ref }} t-\delta_{i}$. This implies that in order to accurately estimate the integral state $z_{i}$, each generator bus needs access only to accurate time and phase measurements, both provided by PMU:s. The decentralized controller architecture is illustrated in figure 3.

Theorem 4. The power system described by (8) where $u_{i}$ is given by (13)-(14), is stable for any choice of $\alpha>0, \beta>0$. Furthermore $\lim _{t \rightarrow \infty} \omega_{i}(t)=\omega^{\text {ref }}$.

Proof: As we are only interested in studying $\omega$, we may rewrite (8) with $u_{i}$ is given by (13)-(14), as

$$
\left[\begin{array}{c}
\dot{z} \\
\dot{\omega}
\end{array}\right]=\underbrace{\left[\begin{array}{cc}
0_{n \times n} & I_{n} \\
-M \mathcal{L}-\beta I_{n \times n} & -M D
\end{array}\right]}_{\triangleq A} \cdot\left[\begin{array}{c}
z \\
\omega
\end{array}\right]+\left[\begin{array}{c}
-\omega^{\mathrm{ref}} \\
M p^{m}
\end{array}\right]
$$

where the $i$ 'th element of $\beta$ is $\beta_{i}$. Let $\underline{m}=\min _{i} 1 / m_{i}$ and $\underline{d}=\min _{i} d_{i}$. We can now write: $M D=\underline{m} \underline{d} I_{n}+D^{\prime}$, where $D^{\prime}$ is a diagonal matrix with non-negative entries. We now consider the the matrix $A^{\prime}$ defined as

$$
A^{\prime} \triangleq\left[\begin{array}{cc}
0_{n \times n} & I_{n} \\
-M \mathcal{L}-\beta I_{n \times n} & -\underline{m} \underline{d} I_{n}
\end{array}\right]
$$

By elementary row and column operations, the eigenvalues of $A^{\prime}$ are given by $\operatorname{det}\left(\left(s^{2}+s \underline{m} \underline{d}\right) I_{n}+M \mathcal{L}+\beta I_{n \times n}\right)$. Comparing this with the characteristic polynomial of $M \mathcal{L}+$ $\operatorname{diag}(\beta)$, we conclude that $s$ must satisfy $s^{2}+s \underline{m} \underline{d}+t_{i}=0$, where $t_{i} \geq 0$ is an eigenvalue of $M \mathcal{L}+\operatorname{diag}(\beta)$. By [18] $M \mathcal{L}+\operatorname{diag}(\beta)$ is positive definite, and hence the above equation has all its solutions in $\mathbb{C}^{-}$. It follows that also $A$ is Hurwitz. Now consider the coordinate shift

$$
\left[\begin{array}{c}
z^{\prime} \\
\omega^{\prime}
\end{array}\right]=\left[\begin{array}{c}
z \\
\omega
\end{array}\right]-\left[\begin{array}{l}
z_{0} \\
\omega_{o}
\end{array}\right]
$$

where $z_{0}=\left(\beta I_{n}+M \mathcal{L}\right)^{-1}\left(M D \omega^{\text {ref }}+p^{m}\right), \omega_{0}=\omega^{\text {ref }} 1_{n \times 1}$. In the translated coordinates, $0_{2 n \times 1}$ is the only equilibrium of the system. Hence $\lim _{t \rightarrow \infty} \omega_{i}(t)=\omega^{\text {ref }} \forall i \in \mathcal{V}$.

\section{Simulations}

The centralized and decentralized frequency control algorithms were tested on the IEEE 30 bus test system [19].The line admittances were extracted from [19] and the voltages were assumed to be $132 \mathrm{kV}$ for all buses. $M$ and $D$ were set to reasonable numerical values. The power system is initially in an operational equilibrium, until the power load is increased by a step of $200 \mathrm{~kW}$ in the buses 2,3 and 7. This will immediately result in decreased frequencies at the extra load buses. The frequency controllers at the buses will then control the frequencies towards the desired frequency of $\omega^{\mathrm{ref}}=50 \mathrm{~Hz}$. In the centralized controller the parameters were set to $\alpha=0.8, \beta=0, \gamma=0.04$, while 
Centralized

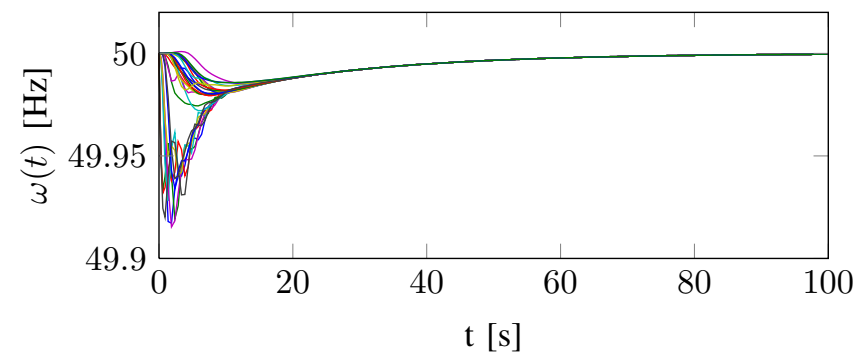

Centralized

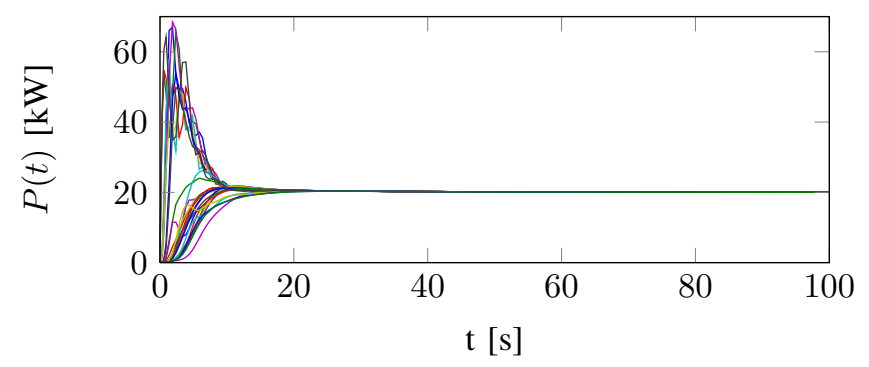

Figure 4. The upper figure shows the bus frequencies with centralized frequency control, while the lower figure shows the control signals at all buses.

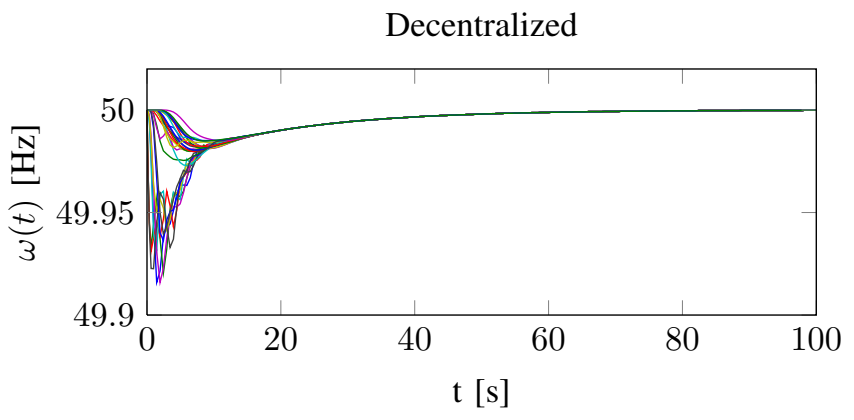

Decentralized

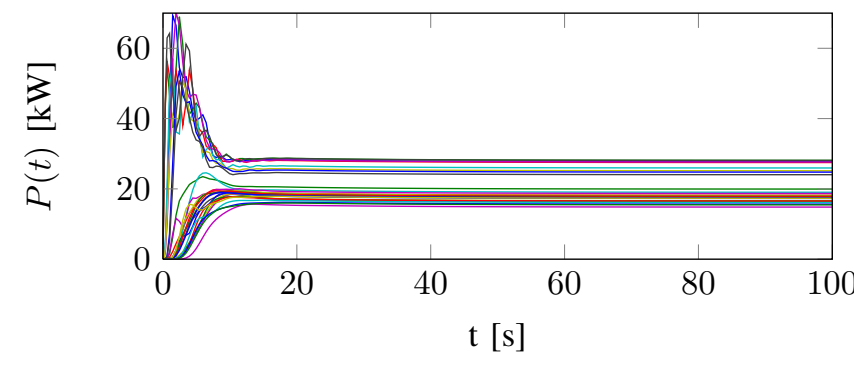

Figure 5. The upper figure shows the bus frequencies with decentralized frequency control, while the lower figure shows the control signals at all buses.

in the decentralized control architecture the parameters were $\alpha=0.8, \beta=0.04$. The step responses of the frequencies is plotted in figures $4-5$. We note that if there is a centralized PI-controller for the reference frequency, the generation is increased uniformly among the generators. If however the integral action is distributed amongst the generators, some generators will increase their generation more than others.

\section{Discussion AND CONCLUSIONS}

In this paper we have studied consensus protocols with integral action for agents with double and single integrator dynamics. We have proved that the proposed consensus protocols reach asymptotic consensus even in the presence of constant disturbances. If we allow for absolute position measurements, the proposed consensus protocol converges asymptotically to a common state. In the absence of disturbances, the proposed consensus protocols asymptotically solve the initial average consensus problem. Furthermore we have demonstrated that a similar consensus protocol can be employed for decentralized frequency control of power systems, with reasonable performance and possible benefits over centralized frequency control.

\section{REFERENCES}

[1] M. Morari and E. Zafiriou. Robust Process Control. Prentice Hall, Englewood Cliffs, 1989.

[2] J. Machowski, J.W. Bialek, and J.R. Bumby. Power System Dynamics: Stability and Control. Wiley, 2008.

[3] N. Senroy, G.T. Heydt, and V. Vittal. Decision tree assisted controlled islanding. Power Systems, IEEE Transactions on, 21(4):1790 -1797, nov. 2006.

[4] B. Yang, V. Vittal, and G.T Heydt. Slow-coherency-based controlled islanding: A demonstration of the approach on the august 14, 2003 blackout scenario. Power Systems, IEEE Transactions on, 21(4):18401847, 2006.

[5] F. Doerfler and F. Bullo. on the critical coupling for Kuramoto oscillators. SIAM Journal on Applied Dynamical Systems, 10(3):1070-1099, 2011.

[6] I Shames, A. M.H. Teixeira, H. Sandberg, and K. H. Johansson Distributed fault detection for interconnected second-order systems. Automatica, 47(12):2757 - 2764, 2011.

[7] R. Olfati-Saber and R.M. Murray. Consensus problems in networks of agents with switching topology and time-delays. IEEE Transactions on Automatic Control, 49(9):1520 - 1533, sept. 2004.

[8] W. Ren and R.W. Beard. Distributed Consensus in Multi-vehicle Cooperative Control. Springer, 2008.

[9] J.A. Fax and R.M. Murray. Information flow and cooperative control of vehicle formations. IEEE Transactions Automatic Control, 49(9):1465 - 1476, sept. 2004.

[10] H.G. Tanner, A. Jadbabaie, and G.J. Pappas. Stable flocking of mobile agents, part i: Fixed topology. In IEEE Conference on Decision and Control, volume 2, pages 2010 - 2015 Vol.2, dec. 2003.

[11] F. Bullo, J. Cortés, and S. Martínez. Distributed Control of Robotic Networks. Applied Mathematics Series. Princeton University Press, 2009.

[12] L. Xiao, S. Boyd, and S. Lall. A scheme for robust distributed sensor fusion based on average consensus. In International Symposium on Information Processing in Sensor Networks, pages 63 - 70, april 2005.

[13] R. Carli, A. Chiuso, L. Schenato, and S. Zampieri. Distributed Kalman filtering based on consensus strategies. IEEE Journal on Selected Areas in Communications, 26(4):622 -633, may 2008.

[14] R.A. Freeman, Peng Yang, and K.M. Lynch. Stability and convergence properties of dynamic average consensus estimators. In IEEE Conference on Decision and Control, pages 338 -343, dec. 2006.

[15] J. R. Silvester. Determinants of block matrices. The Mathematical Gazette, 84(501):pp. 460-467, 2000.

[16] A.G. Phadke. Synchronized phasor measurements in power systems. Computer Applications in Power, IEEE, 6(2):10 -15, april 1993.

[17] R.F. Nuqui and A.G. Phadke. Phasor measurement unit placement techniques for complete and incomplete observability. Power Delivery, IEEE Transactions on, 20(4):2381 - 2388, oct. 2005.

[18] A. Teixeira. Multi-agent systems with fault and security constraints. Master's thesis, Royal Institute of Technology (KTH), June 2009.

[19] Power systems test case archive-30 bus power flow test case. available in: http://www.ee.washington.edu/research/pstca/pf30/pgtca30bus.htm. 